Nloman 2019, 37(2), 53-61

Revista de Psicologia, Ciències de l'Eduació i de l'Esport

ISSN: $1138-3194$

CFacultat de Psicologia, Ciències de l'Educació i de l'Esport Blanquerna

Universitat Ramon Llull

\title{
Evaluación de una experiencia formativa en Flipped Classroom para profesores universitarios de la Universidad Pontificia Comillas de Madrid
}

\author{
Elena Sofía Ojando ${ }^{1}$, Jordi Simón ${ }^{1}$, Miquel Àngel Prats ${ }^{1}$, María Martínez-Felipe ${ }^{2}$, \\ Elsa Santaolalla ${ }^{2} \&$ Jorge Torres ${ }^{2}$ \\ 'Universidad Ramon Llull \\ ${ }^{2}$ Universidad Pontificia Comillas de Madrid \\ Recibido: 2019-6-14 \\ Aceptado: 2019-10-14
}

Evaluación de una experiencia formativa en Flipped Classroom para profesores universitarios de la Universidad Pontificia Comillas de Madrid

\begin{abstract}
Resumen. Este artículo presenta una experiencia de formación en Flipped Classroom dirigida a los docentes universitarios. La experiencia se enmarca en un proyecto estratégico de innovación docente de la Universidad Pontificia Comillas de Madrid que pretende contribuir a la mejora de los procesos de enseñanza y aprendizaje en la universidad sirviéndose de nuevos modelos formativos con el soporte de las TIC. Se describe la implementación de una experiencia de formación sobre el modelo Flipped Classroom dirigida al profesorado universitario y su posterior evaluación a partir de la valoración de los participantes. Para ello, hemos proporcionado un cuestionario al que han respondido un total de 23 profesores universitarios de diferentes titulaciones. Los resultados muestran que el profesorado, aun asistiendo a nuevos modelos formativos con el soporte de las tecnologías digitales, presenta algunas dificultades de tiempos y espacios para diseñar, crear e implementar actividades en el aula. En cambio, el alumnado manifestó interés por disponer de nuevas formas de aprendizaje.
\end{abstract}

Palabras clave: Flipped Classroom; TIC; enseñanza superior

Assessment of a training experience in Flipped Classroom methodology for professors at the Universidad Pontificia Comillas in Madrid

Summary. This article details a training programme in Flipped Classroom methodology offered to university teachers. The training activity was part of a strategic teaching innovation project at the Universidad Pontificia Comillas in Madrid. The aim of the project as a whole was to contribute to the improvement of teaching and learning processes at the university via new educational models using ICT. The article describes the implementation of a training programme in the Flipped Classroom model aimed at university professors and the subsequent assessment of the programme by the participants. Data were collected via a questionnaire that was completed by 23 university professors from a range of disciplines. The results showed that although the professors employ new educational models with the support of digital technologies, they face time and space difficulties in the design, creation and implementation of tasks in the classroom. However, the students were interested in new ways of learning.

Keywords: Flipped Classroom; ICT; Higher education

Correspondence

Elena Sofía Ojando

FPCEE Blanquerna - Universitat Ramon Llull

Email: elenasofiaop@blanquerna.url.edu 


\section{Introducción}

En el contexto actual de la enseñanza superior, donde existe un cambio de paradigma centrado en el aprendizaje del estudiante propio del modelo de enseñanza superior promulgado por Bolonia, se revisan y se actualizan las formas de enseñar en la universidad para tratar de dar respuesta a las nuevas maneras de aprender de las presentes y futuras generaciones de estudiantes. En esta revisión y actualización de los modelos universitarios, desempeñan un papel muy importante las tecnologías de la información y la comunicación (en adelante, TIC) no solo como un recurso sin más, sino como un elemento fundamental dentro de la metodología. De acuerdo con autores como Coll (2010), no se trata de integrar la tecnología en aras de la tecnología, sino de cómo usarla para personalizar de una manera más eficaz el aprendizaje de los estudiantes, teniendo en cuenta que nos hallamos inmersos en una sociedad móvil-céntrica (SMC), es decir, una nueva ecología del aprendizaje en red basada en la influencia masiva de los medios digitales y las TIC en la vida cotidiana. La educación no puede permanecer ajena a los estilos de vida de los estudiantes ni, en este caso, tampoco la universidad. Por el contrario, necesita reconocer estos estilos de vida cambiantes y estar receptiva a ellos en la práctica educativa (González-Patiño \& Esteban-Guitart, 2014).

Esto tiene mayor importancia, si cabe, teniendo en cuenta las características de la generación $\mathrm{Z}$ recopiladas y clasificadas por Fernández-Cruz y Fernández-Díaz (2016), que serían las siguientes: 1) expertos en la comprensión de la tecnología; 2) multitarea; 3) abiertos socialmente desde las tecnologías; 4) rapidez e impaciencia; 5) interactivos; y 6) resilientes. Es preciso recordar que la generación $\mathrm{Z}$ se refiere a alumnos que han nacido entre 1995 y 2012 (Schroer, 2008). Teniendo en cuenta que los alumnos suelen entrar en primer curso de la universidad con 17-18 años, eso implicaría que en los próximos años nuestros alumnos universitarios pertenecerán a esta generación.

Por todo ello, el uso de las TIC es incuestionable no solo porque sería virtualmente imposible que permaneciesen ajenas a la universidad y a la sociedad actual, sino también porque internet y los medios digitales facilitan que cada alumno pueda personalizar el aprendizaje de modo que aprenda a su propio ritmo y se convierta en ciudadano digital responsable en un futuro próximo. Además, le ayudan a profundizar su aprendizaje. El NMC Horizon Report, concretamente en la edición sobre educación superior del 2016, señala como un reto la personalización del aprendizaje, por lo que hay que implementar modelos pedagógicos que lo permitan.

Es en este contexto, en el que la tecnología está presente y las necesidades de los estudiantes se dirigen hacia nuevas formas de aprender, donde aparecen nuevos modelos de enseñanza y aprendizaje como el Flipped Classroom (Bergmann \& Sams, 2012), en el que el enfoque se centra en el estudiante y en ofrecerle oportunidades de aprendizaje significativo durante su formación inicial en la universidad, ya que, tal y como apunta Marc Prensky en el prólogo del libro de Bergmann y Sams (2012), "el viejo papel de transmitir información ha sido sustituido por enseñar a los estudiantes a enseñarse a sí mismos". Por lo tanto, no se trata solamente de ofrecer conocimientos a los estudiantes, sino también de ofrecer oportunidades de aprendizaje que permitan desarrollar las competencias propias de su rol profesional.

El modelo Flipped Classroom surge en el 2006 en un instituto de secundaria de Colorado (EE. UU.) de la mano de Bergmann y Sams, que, para poder cubrir las necesidades de sus alumnos, empezaron a grabar sus sesiones para que los estudiantes pudieran acceder a sus explicaciones y dedicar el tiempo en clase a ayudarles con las dudas o prácticas que había que realizar. Finalmente, en el 2007 acuñan el nombre Flipped Classroom (Bergmann \& Sams, 2012).

En esencia, el modelo Flipped Classroom plantea un trabajo autónomo previo a la clase por parte del estudiante que accede a las explicaciones del profesor y un trabajo en clase para poder resolver dudas e incluso poner en práctica la teoría aprendida previamente con la ayuda del profesor/a y de sus compañeros/as. No hay una sola forma de dar la vuelta a la clase, tal y como explican los mismos autores. No existe ninguna metodología concreta que se haya de reproducir paso a paso para poder dar la vuelta a las clases, sino que es más bien un cambio de enfoque centrado en el estudiante y su aprendizaje (Bergmann \& Sams, 2014).

A partir de ahí, cada profesor puede dar la vuelta a sus clases de diferente manera, aunque teniendo en cuenta la esencia del modelo, en el que existe un espacio de aprendizaje autónomo por parte del estudiante y un espacio de aprendizaje colaborativo en el aula. En ese punto nos encontramos con dos escenarios propios de la enseñanza superior (trabajo autónomo y trabajo colaborativo), y es donde el modelo Flipped Classroom encaja como modelo de enseñanza y aprendizaje en la formación inicial de los futuros profesionales. No solamente por este enfoque en el que se establecen dos escenarios, sino también por el enfoque centrado en el desarrollo de competencias de los estudiantes y en la personalización del aprendizaje (Bergmann \& Sams, 2014).

Es por todo ello que presentamos esta propuesta de formación al profesorado universitario, para que puedan dar la vuelta a sus clases, ofreciendo a sus estudiantes escenarios de aprendizaje real que les permitan desarrollar su autonomía, compromiso y responsabilidad, así como las competencias propias de su ámbito profesional.

Por lo tanto, el objetivo de este artículo es evaluar una propuesta de formación en Flipped Classroom dirigida al profesorado universitario de diferentes estudios de la Universidad Pontificia Comillas de Madrid según la percepción de los participantes y datos recogidos a partir de la implementación en sus clases. 


\section{Contexto de desarrollo del proyecto}

Esta experiencia ha formado parte de un proyecto de formación permanente del profesorado universitario y de innovación docente que se ha llevado a cabo en la Universidad Pontificia Comillas de Madrid, a lo largo de estos tres últimos cursos, bajo el modelo Flipped Classroom impartido por docentes de la FPCEE Blanquerna - Universitat Ramon Llull. Plantearemos las bases que justifican y sostienen el desarrollo de esta experiencia de formación, así como sus características definitorias, el proceso de implementación y un análisis de su impacto y sus resultados más significativos.

El proyecto de formación del profesorado universitario y de innovación docente, en el que se enmarca esta experiencia de formación, encuentra su justificación en el marco de lo establecido en el Plan Estratégico de la Universidad para el período 2014-2018. En este plan se parte de la base de que una universidad del siglo XXI ha de ser una "learning institution", una institución de conocimiento y aprendizaje continuo a través de la investigación-innovación y de la enseñanza en actualización permanente y profunda apertura y compromiso con la realidad social (p. 6).

Además, a lo largo de los últimos años, en el marco del proceso de implantación del nuevo Espacio Europeo de Educación Superior (EEES), la Universidad ha recorrido un intenso trayecto en la adecuación y adaptación de nuestros sistemas docentes a las nuevas posibilidades pedagógicas. Partiendo de esta base, y ante el análisis realizado sobre las necesidades actuales y el nivel de competencia del profesorado, desde el Instituto de Ciencias de la Educación (ICE) de la universidad planteamos este proyecto de formación en el que la Flipped Classroom y el uso de las TIC toman protagonismo para responder a la dimensión estratégica del área 1 sobre el modelo formativo, que trata sobre la consolidación de una oferta excelente de títulos con entidad e identidad, equilibrada y especializada, ordenada y viable, conectada internacional, profesional y académicamente.

Esta experiencia formativa tiene tres características básicas que la definen y la fundamentan:

- Flexibilidad; la propuesta fue cambiando y adaptándose a la luz de los resultados obtenidos y de las nuevas necesidades y demandas detectadas.

- Capacidad de adaptación a la realidad específica de la universidad, centrándose en las necesidades detectadas en el profesorado y partiendo de las herramientas TIC institucionales de las que la universidad se ha dotado para mejorar su funcionamiento interno y su oferta y metodología formativa.

- Sostenibilidad, por lo que no pretende ser una oferta extensa de actividades formativas, sino que se centró en las necesidades y medios más importantes y relevantes. Por ello es una propuesta de carácter más bien reducido.

El desarrollo de los talleres se implementó siguiendo las características propias del modelo Flipped Classroom y con el apoyo de las TIC; de ese modo, los profesores participantes podían experimentar en primera persona el aprendizaje a través de este nuevo enfoque. Por eso, hubo una parte de trabajo autónomo no presencial y una parte de trabajo autónomo presencial.

Al finalizar el taller se espera que los participantes sean capaces de diseñar e implementar una clase inversa utilizando diferentes herramientas TIC y recursos digitales 2.0 respondiendo a los objetivos siguientes:

1. Identificar el modelo Flipped Classroom y sus características específicas.

2. Diseñar e implementar una sesión de clase siguiendo el modelo Flipped Classroom (clase inversa).

3. Seleccionar diferentes herramientas y recursos digitales web 2.0 y apps de diferentes temáticas para su uso en el modelo Flipped Classroom.

4. Conocer diferentes experiencias de Flipped Classroom en la educación superior.

5. Crear una propuesta didáctica bajo el modelo Flipped Classroom.

La implementación se dividió en tres partes: una primera sesión para poder presentar el taller y las tareas previas a la sesión presencial; una sesión presencial, donde se invirtió la mayoría del tiempo del taller en diferentes actividades bajo metodologías activas; y después del taller, para que los participantes pudieran diseñar su primera clase inversa y hacer la entrega a través de la plataforma Moodle.

En la tabla 1 podemos observar la descripción de cada una de las actividades propuestas a los participantes en el taller.

Al finalizar el taller, los participantes disponían de todo el material sobre Flipped Classroom para realizar su propia clase inversa, así como una infografía que explica detalladamente las fases y etapas para dar la vuelta a las clases (Prats y Ojando, 2017, pp. 110-114).

\section{Método}

El presente artículo hace referencia a la evaluación de la experiencia de formación al profesorado universitario en Flipped Classroom según la percepción de los participantes y datos recogidos a partir de la implementación en sus clases. Por lo tanto, la investigación se basa en una metodología mixta (cualitativa y cuantitativa) con el fin de analizar la valoración de la experiencia de formación y resultados en la docencia universitaria.

Desde el punto de vista metodológico, la dicotomía cuantitativo-cualitativa muestra uno de sus efectos nocivos en la ocultación de otras posibilidades metodológicas. Dicha dicotomía no solo ha operado en la comunidad científica a lo largo del siglo XX ofreciendo respectivos procesos, sino que los defensores de la teoría de la incompatibilidad entre los paradigmas los presentan como ofertas excluyentes, separadas en compartimentos estancos, opuestos. En la actualidad, autoras como Anguera (2010) abogan por la complementariedad metodológica, aunque existan problemas, salvables según ella, a lo largo de todo el proceso. Así, 
Tabla 1. Propuesta de actividades del taller de la clase inversa

\begin{tabular}{|c|c|}
\hline Fases del taller & Actividades dirigidas por los formadores \\
\hline $\begin{array}{l}\text { Presentación } \\
\text { Previa a la sesión }\end{array}$ & $\begin{array}{l}\text { Mensaje de bienvenida. } \\
\text { Presentación de una guía del curso. } \\
\text { Primeras orientaciones para el trabajo previo a la sesión. }\end{array}$ \\
\hline Tareas previas a la sesión & $\begin{array}{l}\text { Tareas a realizar durante una semana antes de la formación presencial: } \\
\text { - presentación de los asistentes en un Padlet } \\
\text { - participación en un formulario de conocimientos previos sobre el modelo Flipped Classroom }\end{array}$ \\
\hline Aproximación teórica previa a la sesión & $\begin{array}{l}\text { Visualización de un vídeo sobre el modelo Flipped Classroom a través de la herramienta edpuzzle con el fin de } \\
\text { tener un seguimiento del trabajo autónomo de los participantes al taller y responder a preguntas clave sobre el } \\
\text { propio vídeo. }\end{array}$ \\
\hline Presentación en clase & $\begin{array}{l}\text { Presentación de los asistentes - Desvirtualización. } \\
\text { Presentación de los resultados del cuestionario de conocimientos previos sobre Flipped Classroom. } \\
\text { Revisión de visualización del vídeo y resultados en las respuestas. }\end{array}$ \\
\hline $\begin{array}{l}\text { Cuestionario inicial } \\
\text { Con kahoot }\end{array}$ & $\begin{array}{l}\text { Dinamizar un Kahoot (pocas preguntas y crear debate con los asistentes). } \\
\text { Evaluar los contenidos aprendidos a través del vídeo. } \\
\text { Aprender a crear un Kahoot y dinamizarlo. }\end{array}$ \\
\hline $\begin{array}{l}\text { Ronda de preguntas, dudas y debate } \\
\text { abierto }\end{array}$ & $\begin{array}{l}\text { Resolver dudas sobre el modelo Flipped Classroom. } \\
\text { Preguntas que debemos hacernos antes de diseñar nuestra primera clase inversa. } \\
\text { Compartir experiencias entre los docentes participantes. }\end{array}$ \\
\hline $\begin{array}{l}\text { Creación de grupos } \\
\text { De trabajo } \\
\text { (Dos propuestas) }\end{array}$ & $\begin{array}{l}\text { 1. a propuesta: se distribuyen los participantes para trabajar en grupo con la finalidad de que descubran los } \\
\text { diferentes pasos para dar la vuelta a la clase. } \\
\text { 2. }{ }^{\text {a }} \text { propuesta: se distribuyen los participantes para trabajar en grupo con la finalidad de que descubran } \\
\text { diferentes herramientas } 2.0 \text { que se utilizan en la clase inversa. } \\
\text { En las dos propuestas, cada grupo trabaja en una diapositiva de una presentación compartida. }\end{array}$ \\
\hline $\begin{array}{l}\text { Puesta en común } \\
\text { Por grupos }\end{array}$ & $\begin{array}{l}\text { Cada grupo expone los aprendizajes realizados y los comparte con el resto de los grupos de trabajo. Todo ello se } \\
\text { comparte en una misma presentación y, a la vez, se van resolviendo las dudas. }\end{array}$ \\
\hline $\begin{array}{l}\text { Tareas a realizar } \\
\text { Después del taller } \\
\end{array}$ & $\begin{array}{l}\text { Tarea a realizar: diseñar su primera clase inversa. } \\
\text { Presentación de la plantilla para diseñar su primera clase inversa. }\end{array}$ \\
\hline Valoración del taller & Realizar una valoración de la formación recibida en el taller para finalizar la sesión. \\
\hline
\end{tabular}

y siguiendo a Hernández Sampieri y Mendoza (2008), la metodología mixta representa un conjunto de procesos sistemáticos, empíricos y críticos de investigación que implican la recopilación y el análisis de datos cualitativos y cuantitativos, así como su integración y discusión conjunta, para obtener inferencias de toda la información recabada y lograr un mayor entendimiento del fenómeno bajo estudio.

Por su parte, autores como Hernández Sampieri y Mendoza (2008), Bryman (2008), Hernández et al. (2014) y Delgado Álvarez (2014) describen las ventajas de los enfoques metodológicos mixtos: las relaciones entre investigador, fenómeno y participantes están determinadas en función de cada estudio en particular y por el contexto; rechazan la dicotomía entre dualismos convencionales y se orientan más a la acción que a la discusión filosófica.

La experiencia de formación se realizó en cuatro ediciones distintas. En cada edición hubo un número de participantes diferente.

Tabla 2. Profesores de la Universidad Pontificia Comillas de Madrid que participaron en los talleres de Flipped Classroom

\begin{tabular}{ll}
\hline Ediciones & Número de participantes \\
\hline Edición 1 (2014/15) & 24 \\
\hline Edición 2 (2015/16) & 17 \\
\hline Edición 3 (2016/17) & 8 \\
\hline Edición 4 (2017/18) & 15 \\
\hline Total & 64 \\
\hline
\end{tabular}

\section{Instrumentos}

La recogida de datos se hizo a través de un cuestionario rellenado por cada participante de las diferentes ediciones. El cuestionario se administró en línea utilizando formularios de Google y era anónimo. Consistía en un total de 34 preguntas, de las cuales 22 eran de res- puesta cerrada y 12 de respuesta abierta, respondiendo a las variables de análisis siguientes:

A. Datos demográficos

B. Experiencias previas con Flipped Classroom

C. Aplicación del modelo Flipped Classroom en la docencia universitaria

D. Cambios en los estudiantes

E. Cambios en la práctica docente

F. Valoración de la formación

\section{Participantes}

Del total de 64 participantes en los talleres, respondieron un total de 23 profesores de la Universidad Pontificia Comillas de Madrid (España), configurando así la muestra de este estudio. El rango de edad de la muestra se sitúa entre 32 y 63 años, con $\bar{\chi}=46.30$ y una moda de 54 años de edad. La representación por sexos es de $60.9 \%$ mujeres y $39.1 \%$ hombres. Por lo tanto, la mayoría del profesorado participante en el estudio eran mujeres y representaban a diferentes disciplinas científicas, tal y como se puede observar en el gráfico siguiente.

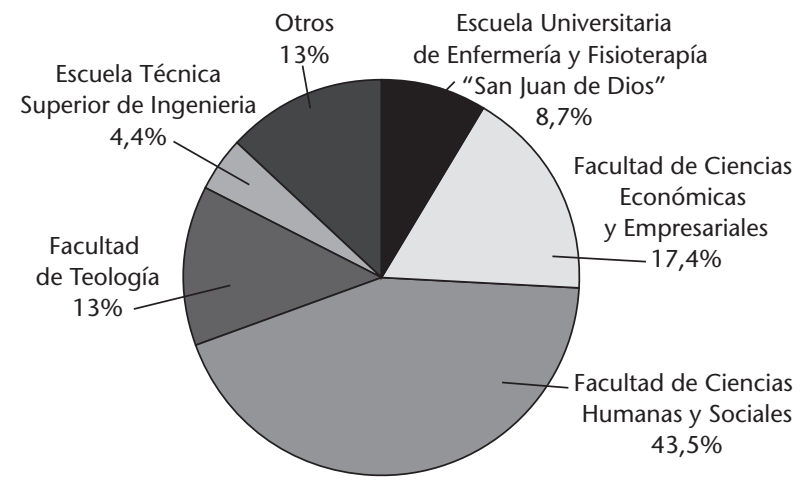

Figura 1. Centros de procedencia del profesorado (Universidad Pontificia Comillas de Madrid, 2017) 
Siendo el centro predominante, con un $43.5 \%$, la Facultad de Ciencias Humanas y Sociales, donde se cursan, por ejemplo, los grados de Educación, de Actividad Física y Deporte, de Filosofía, etc.

\section{Procedimiento}

El análisis de los resultados extraídos de las respuestas al cuestionario se ha realizado a partir de la aplicación de estadística descriptiva. Por lo tanto, se ha tratado el conjunto de los datos obtenidos sin diferenciar entre los participantes de las diversas ediciones, ya que han realizado la misma formación (igual estructura, dinámicas y equipo de formadores). De ese modo, se han seguido las siguientes fases:

Fase 1: diseño y validación del cuestionario

Fase 2: envío del cuestionario en línea, realizado con

Google Forms (y recordatorios pertinentes)

Fase 3: recogida de datos y análisis de datos realizados

con SPSS y Google Sheet

Fase 4: descripción de los resultados

En relación con las preguntas abiertas, se ha llevado a cabo un análisis cualitativo basado en procesos de codificación mixta partiendo de las categorías previas de análisis e identificando nuevas categorías en cada una de las respuestas.

\section{Resultados y discusión}

A continuación presentamos los resultados derivados de las respuestas de los participantes siguiendo el esquema marcado por el cuestionario de recogida de datos:

A. Experiencias previas con Flipped Classroom

B. Aplicación del modelo Flipped Classroom en la docencia universitaria

C. Cambios en los estudiantes

D. Cambios en la práctica docente

E. Valoración de la formación

\section{A. Experiencias previas con Flipped Classroom}

Del conjunto de profesores que respondieron el cuestionario de recogida de datos, aproximadamente casi la mitad de ellos ya conocían la existencia del modelo Flipped Classroom (47.8\%), sobre todo a través de páginas web (58.3\%) o algún compañero/a de la universidad (16.7\%). Por otro lado, el 73.9\% afirmó que no había experimentado anteriormente con el modelo Flipped Classroom, y solo un $21.7 \%$ dijo haberlo utilizado como profesor.

Por lo tanto, dado que los participantes tenían algunos conocimientos previos sobre Flipped Classroom, en todas las ediciones se pasó, antes de la sesión presencial, un cuestionario de conocimientos previos para así poder adaptar mucho mejor la sesión y reflejar una de las características propias de esta propuesta, la flexibilidad.

\section{B. Aplicación del modelo Flipped Classroom en la docencia universitaria}

Los profesores participantes en las formaciones y que respondieron el cuestionario constataron que, después de haber realizado el taller de Flipped Classroom, habían podido aplicarlo en sus clases. Concretamente, un $17.4 \%$ de los profesores "voltearon" totalmente sus clases, un $65.2 \%$ aplicaron parcialmente los aprendizajes recibidos y solo un $17.4 \%$ no lo hicieron.

Los profesores que respondieron negativamente, sobre todo hicieron referencia a cuestiones técnicas y no pedagógicas que les impedían cambiar de estilo docente. Por ejemplo, adujeron falta de tiempo por motivos familiares $\mathrm{u}$ otros para dedicarlo a la preparación, tener demasiados estudiantes, etc. Asimismo, una persona señaló que no lo había implementado por "la inseguridad sobre el resultado".

En cuanto a las herramientas utilizadas en la implementación del modelo Flipped Classroom, nos encontramos con una variedad importante.

En la figura 2 vemos el predominio de los vídeos como herramienta utilizada antes de la sesión presencial. En la categoría "otros", encontraríamos herramientas como Socrative, TESTeach, SR2.0, ThingLink, Blog de aula, SlideShare, EduCanon, Twitter.

Cuando la misma pregunta se refiere a las herramientas utilizadas en el momento presencial del modelo Flipped Classroom, encontramos menos respuestas y las herramientas predominantes son las que permiten recoger al momento datos de los estudiantes, siendo la más mencionada (7 ocasiones) Kahoot.

En este apartado también se preguntó sobre las asignaturas en las que se implementó el modelo Flipped Classroom. Siguiendo la pauta marcada por la diversa procedencia de los profesores, las respuestas también son muy diferentes: Ética de la Praxis Sanitaria, English for Business Studies, Terminología, Retórica Persuasiva y Oratoria RICOM, Gobierno Corporativo, Consumo Responsable, Interpretación I, Actualización Científica y Currículo en Matemáticas, Comunicación Audiovisual y Multimedia, etc. Los cursos de implementación oscilaron entre primero y cuarto e impactaron en unos 1.200 estudiantes de la Universidad Pontificia de Comillas.

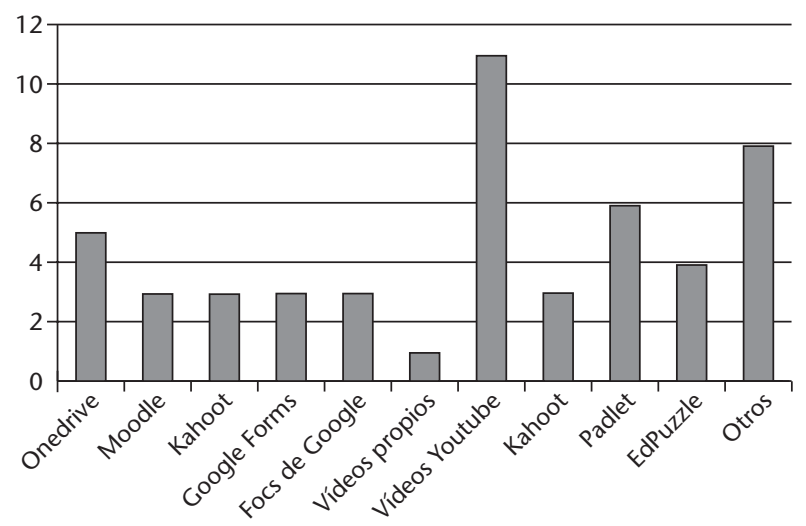

Figura 2. A la hora de implementar el modelo Flipped Classroom escribe qué herramientas digitales utilizaste antes de la sesión (Universidad Pontificia Comillas de Madrid, 2017) 
En varias actividades puntuales

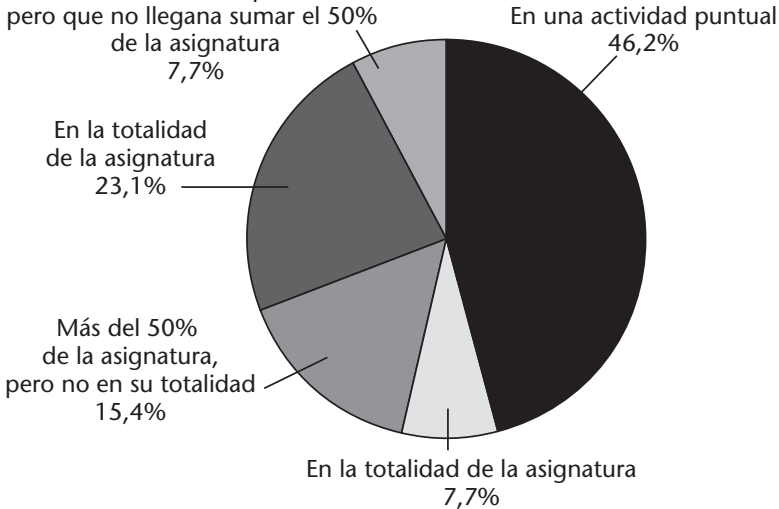

Figura 3. Nivel de aplicación del modelo Flipped Classroom en colaboración con otros profesores (Universidad Pontificia Comillas de Madrid, 2017).

También se preguntó por la colaboración entre profesores en la aplicación del modelo Flipped Classroom. Como nota destacada, un $41.7 \%$ de los profesores mencionaron que trabajaron colaborativamente, pero solo de manera puntual, tal y como se puede observar en la figura 3.

\section{Cambios en los estudiantes}

Las tres primeras preguntas de este apartado hacían referencia a la mejora de diversos aspectos relacionados con los estudiantes, siendo 1 la valoración que indicaba poco cambio y 4 la que indicaba mucho cambio. Los resultados fueron los siguientes:

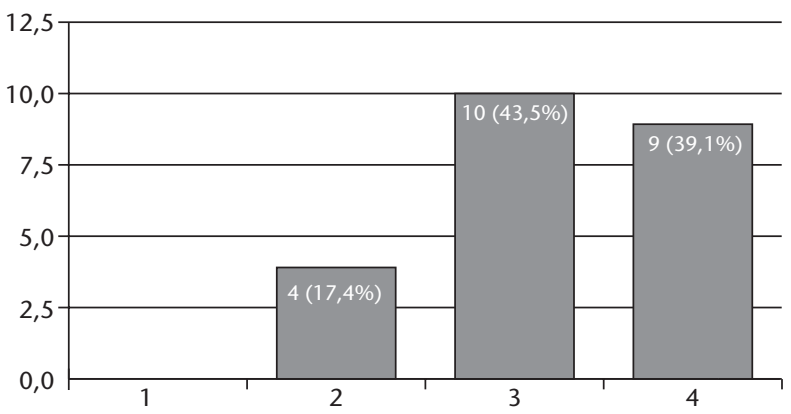

Figura 4. ¿Ha mejorado la implicación de tus alumnos en tu materia? (Universidad Pontificia Comillas de Madrid, 2017)

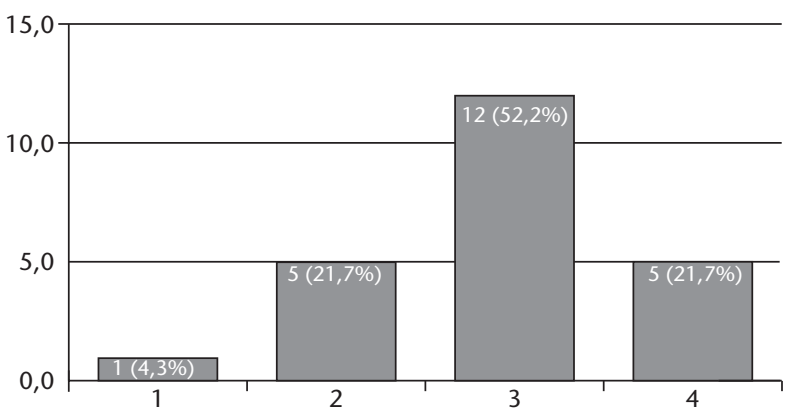

Figura 5. ¿Han mejorado los resultados académicos de tus alumnos al realizar este tipo de actividades? (Universidad Pontificia Comillas de Madrid, 2017)

Los dos gráficos muestran una mejora en los diferentes aspectos por parte de los estudiantes. Cabe re- saltar las mejoras en los aspectos motivacionales y, en menor medida, en los rendimientos académicos.

Otros aspectos relacionados con los cambios en los estudiantes indican que aceptaron mayoritariamente (60.9\%) la nueva propuesta metodológica y que no supuso conflicto alguno para ellos (95\%).

Podemos ver, por lo tanto, que se cumple la observación de Bergmann y Sams (2014) cuando describen el modelo Flipped Classroom como un cambio de enfoque centrado en el estudiante y su aprendizaje y no tanto como una metodología a seguir.

Los profesores señalan, además, que en la implementación del Flipped Classroom han percibido que los alumnos experimentan algunos cambios:

- Curiosidad por la asignatura

- Interés

- En algunos casos se divierten más

- Mucha participación en clase y a través de la plataforma Moodle

- Más colaboración entre ellos, más socialización

- Motivación e implicación

- Piden que se extiendan las sesiones con Flipped Classroom durante todo el cuatrimestre

- La motivación por la asignatura, la asistencia, los resultados de actividades que antes eran no presenciales y ahora se realizan de manera colaborativa en el aula, el proceso de resolver dudas

Aunque hay que destacar también algunas valoraciones que hacen referencia a la falta de cambios: "Los cambios siempre cuestan y siempre te responden que no tienen tiempo para ver con antelación los contenidos; algunas veces los alumnos suelen sugerir más clase magistral y 'ordenada' según sus estándares, porque 'tanta innovación llega a marear'”.

\section{Cambios en la práctica docente}

Preguntamos a los profesores sobre los cambios en la práctica docente a partir de una batería de diez preguntas en que tenían que valorar sus prácticas docentes en un rango de 1 a 4 , en el que 1 era nada y 4 mucho. Los resultados fueron los que muestra la tabla 3 .

De este conjunto de datos se puede destacar la $\bar{\chi}=3.1$, próximo al valor "Bastante", con lo que podríamos decir que, en general, los profesores se sentirían bastante cercanos a muchas de las características que definen a un profesor que implementa el modelo Flipped Classroom.

De esta matriz de datos cabe destacar que son los ítems con una $\bar{\chi}=3.3$. Así, tenemos profesores que se preocupan por favorecer los trabajos autónomos, que ponen al alumno en el centro del sistema y que crean marcos para poder interactuar. También destacan los profesores que consideran que están a disposición del estudiante para dar feedback cuando sea necesario, con una media de 3.7 .

En este sentido, tal y como apuntan Bergmann y Sams (2012), el modelo Flipped Classroom permite dedicar más tiempo para poder proporcionar feedback a los estudiantes de forma continuada durante la clase. 
Tabla 3. Valora en qué medida te identificas con estas afirmaciones (Universidad Pontificia Comillas de Madrid, 2017)

\begin{tabular}{|c|c|c|c|c|c|}
\hline & Nada (\%) & Poco $(\%)$ & Bastante (\%) & Mucho (\%) & $\bar{\chi}$ \\
\hline $\begin{array}{l}\text { Creo marcos de espacio y tiempo que permiten a los estudiantes interactuar y reflexionar } \\
\text { sobre su aprendizaje. }\end{array}$ & 0 & 1 & 14 & 8 & 3.3 \\
\hline $\begin{array}{l}\text { Observo y hago seguimiento a los estudiantes para hacer ajustes o dar feedback cuando es } \\
\text { necesario. }\end{array}$ & 0 & 4 & 10 & 9 & 3.2 \\
\hline $\begin{array}{l}\text { Ofrezco a los estudiantes diversas oportunidades de involucrarse en actividades } \\
\text { significativas en las que el profesor no es la pieza central. }\end{array}$ & 0 & 3 & 10 & 10 & 3.3 \\
\hline $\begin{array}{l}\text { Estructuro y guío estas actividades y las hago accesibles a todos los estudiantes a través } \\
\text { del feedback y una adaptación personalizada. }\end{array}$ & 0 & 5 & 13 & 5 & 3.0 \\
\hline Priorizo los contenidos para favorecer el trabajo autónomo de los estudiantes. & 0 & 2 & 13 & 8 & 3.3 \\
\hline $\begin{array}{l}\text { Selecciono o diseño materiales para desarrollar contenidos relevantes — por lo general } \\
\text { material multimedia - para mis estudiantes. }\end{array}$ & 0 & 5 & 10 & 8 & 3.1 \\
\hline Diferencio distintos niveles de complejidad para atender la diversidad de mis estudiantes. & 1 & 8 & 11 & 3 & 2.7 \\
\hline $\begin{array}{l}\text { Estoy a disposición de los estudiantes para dar feedback individual o grupal inmediato } \\
\text { según sea requerido. }\end{array}$ & 0 & 1 & 6 & 16 & 3.7 \\
\hline $\begin{array}{l}\text { Llevo a cabo evaluaciones formativas durante el tiempo de clase a través de la } \\
\text { observación y el registro de información para complementar la instrucción. }\end{array}$ & 3 & 3 & 13 & 4 & 2.8 \\
\hline $\begin{array}{l}\text { Colaboro y reflexiono con otros profesores y asumo la responsabilidad de la } \\
\text { transformación de mi práctica docente. }\end{array}$ & 2 & 9 & 5 & 7 & 2.7 \\
\hline
\end{tabular}

En la franja más baja hay tres medias que no llegan a 3 puntos (bastante), y son los ítems que hacen referencia a la implementación de evaluaciones formativas $\mathrm{y}$ al hecho de tener diferentes niveles para atender la diversidad en el aula. En estos dos ítems hay profesores que declaran que no lo hacen nunca. Lo mismo podríamos decir del ítem que hace referencia a la colaboración entre profesores.

A continuación, se le ofrecía al profesor responder a una pregunta abierta para poder completar estos datos, y concretamente se le preguntaba sobre si había algún otro aspecto de su docencia que hubiera cambiado.

De las múltiples respuestas obtenidas podemos destacar las que hacen referencia al hecho de que los profesores han tenido que aumentar sus capacidades en la planificación docente, lo que, al final, entienden como una mejora. Destacamos la siguiente respuesta:

"Creo que planificar sesiones con Flipped Classroom me obliga constantemente a ponerme en el papel del estudiante y salir del rol cómodo del profesor que imparte clases de tipo magistral; de esta manera creo que los 'humanizo' y resulta más sencillo empatizar con ellos, ver dónde están los problemas de la asignatura, qué cosas se pueden explicar o trabajar mejor, etc.".

También hay respuestas relativas a un mayor atractivo del proceso de enseñanza/aprendizaje, lo que también repercute en ellos como docentes. Por ejemplo:

"Con la respuesta positiva de los estudiantes yo me siento más motivado para seguir innovando". Asimismo, diversos profesores señalaron que la aplicación del modelo Flipped Classroom les permitía estar actualizados en cuestiones tecnológicas.

Para acabar este bloque dedicado a la docencia, se les preguntaba por las dificultades en cuanto a la implementación del modelo Flipped Classroom. Las respuestas giraron en torno principalmente a la falta de tiempo y el exceso de planificación docente:

"Lo laborioso que resulta elaborar los contenidos con antelación y la necesidad de una planificación casi milimétrica de la asignatura en su conjunto y de cada sesión individual; pero no lo considero tanto un 'obstáculo' como un mal necesario que, $a$ posteriori, facilita muchísimo el trabajo".

"[...] el tiempo extra que requiere la planificación de una o varias sesiones Flipped".

"Rechazo inicial por falta de tiempo".

Los profesores también manifestaron dificultades tecnológicas en dos respuestas.

\section{E. Valoración de la formación}

Este bloque de datos consiste en una pregunta cerrada que, como en otros casos, tiene un rango entre 1-4 y una serie de preguntas abiertas en las que se profundiza en la valoración. Concretamente, la pregunta ("En definitiva, teniendo en cuenta la formación recibida en el ICE, ¿cómo ha sido la experiencia?") tuvo una $\bar{\chi}=3.7$, cerca del máximo.

Las respuestas obtenidas a la pregunta abierta (“¿Qué necesitarías para seguir avanzando o que otros siguieran avanzando?") se clasificaron en tres categorías, que son, por orden de importancia, las siguientes: 1. Necesito más formación para seguir avanzando, con nueve respuestas como:

- Más cursos con ejemplos de aplicación concretos.

- Cursos orientados a la psicología del alumno, lenguaje no verbal, control de las emociones de los alumnos.

- Continuar conociendo nueva formación para seguir avanzando.

2. Cuestiones relativas a la estructura académica y los recursos. Con cuatro respuestas, las cuales las incluimos todas, ya que son significativas y muy diferentes las unas de las otras:

- "Poder usar los recursos de la universidad. Por ejemplo, los vídeos utilizados los he grabado yo en mi propio ordenador de una forma 'artesanal' o 'casera'; creo que serían susceptibles de mejora cualitativa con los medios de la Universidad".

- "Que hubiera más flexibilidad con los horarios para compartir docencia con otros profesores y poder llevar a cabo actividades colaborativas en espacio-tiempo real". 
Tabla 4. Puntos fuertes de la formación recibida (Universidad Pontificia Comillas de Madrid, 2017)

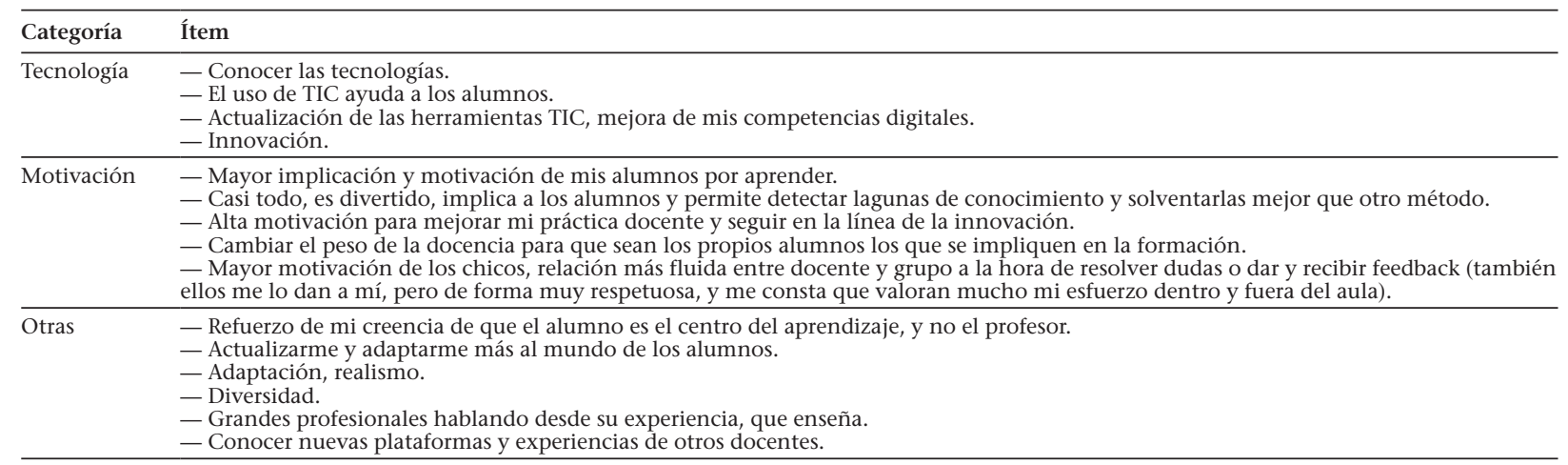

Tabla 5. Puntos débiles de la formación recibida (Universidad Pontificia Comillas de Madrid, 2017)

\begin{tabular}{|c|c|}
\hline Categoría & Ítem \\
\hline Tiempo & $\begin{array}{l}\text { - Falta tiempo para la preparación de las asignaturas con el costo que precisan. } \\
\text { - Falta de tiempo para seguir avanzando. } \\
\text { - Que es muy condensado, a veces muchas horas y a veces no se dan muchas plazas. } \\
\text { - Aunque en internet se puede encontrar de todo, algunos vídeos para determinados temas son muy malos, y yo reconozco que salir en } \\
\text { los mismos y realizarlos implica mucho tiempo. }\end{array}$ \\
\hline Formación & $\begin{array}{l}\text { - Necesitaría mejorar mi formación y convencer al resto del equipo de formación sobre su utilidad. } \\
\text { - Como todo, iré afinando las asignaturas con cada curso, pero la experiencia es positiva y permite personalizar el aprendizaje. } \\
\text { Probablemente tenga que mejorar la evaluación modificando los porcentajes de cada tarea. } \\
\text { - Perfeccionar la metodología; en algunos casos muy aislados, la percepción de algunos estudiantes es que la asignatura es "un juego" o } \\
\text { "una chorrada", como que al ludificar el aprendizaje se banaliza... Afortunadamente son los menos. } \\
\text { - Tengo que ampliar mi formación en informática. }\end{array}$ \\
\hline $\begin{array}{l}\text { Dinámica del } \\
\text { curso }\end{array}$ & $\begin{array}{l}\text { - Hay un flujo excesivo de trabajo para el estudiante para poder desarrollar las distintas actividades de forma efectiva. } \\
\text { - Del curso uno no se imagina de verdad lo que suponen la implantación y sus consecuencias. } \\
\text { - Excesiva presentación de herramientas. } \\
\text { — Las herramientas cambian fácilmente y requieren tiempo de autoformación. }\end{array}$ \\
\hline Otras & $\begin{array}{l}\text { - Sensación de no llegar a todo y una cierta "frustración" por no poder incorporar todo lo aprendido. Creo que tampoco s } \\
\text { suficiente por parte del departamento, facultad y universidad estos esfuerzos personales para la mejora de la práctica docen }\end{array}$ \\
\hline
\end{tabular}

- "Motivación extrínseca, ya que de la intrínseca ando sobrada. Me da la impresión de que no se valora la innovación docente a la hora de ascender".

- "Un seguimiento más personalizado de la adaptación del profesorado a estos nuevos modelos de enseñanza".

3. Para poder seguir avanzando, necesito más tiempo (tres respuestas), con aportaciones como:

- Más tiempo para poder prepararme mejor las clases.

- Más tiempo "libre" que poder invertir en pensar y crear nuevos materiales.

Para finalizar las valoraciones, los profesores de la Universidad Pontificia Comillas que participaron en programas de formación sobre Flipped Classroom fueron preguntados sobre los puntos fuertes y las debilidades de la formación (tablas 4-5).

De ese modo, podemos observar que los participantes destacan sobre todo como puntos fuertes el desarrollo de la competencia digital y el uso de las TIC en sus clases, la motivación que perciben por parte de sus estudiantes y la capacidad de adaptación a las nuevas formas de aprendizaje. Por otro lado, destacan como puntos débiles la falta de tiempo para poder transferir a sus clases todo lo aprendido, la necesidad de seguir formándose y el exceso de herramientas.

\section{Conclusiones}

Tal y como se ha comentado con anterioridad, desde el Instituto de Ciencias de la Educación (ICE) de la
Universidad Pontificia Comillas se planteó una propuesta formativa básica para responder a la dimensión estratégica del área 1 sobre el modelo formativo, que trata sobre "la consolidación de una oferta excelente de títulos con entidad e identidad, equilibrada y especializada, ordenada y viable, conectada internacional, profesional y académicamente". En este sentido, las conclusiones que presentamos van a responder a cada uno de estos siguientes objetivos operativos:

1. Aumento de la utilización de las TIC avanzadas en la metodología pedagógica universitaria de todas las áreas de actividad docente.

2. Implementación en mayor grado de una metodología participativa y cooperativa de aprendizaje.

En cuanto al aumento de la utilización de las TIC avanzadas en la metodología pedagógica universitaria, de los datos obtenidos, analizados e interpretados podemos concluir lo siguiente:

1. Los profesores confirman y señalan que conocen nuevas metodologías docentes, aunque no las aplican por no disponer de las habilidades tecnológicas suficientes ni del tiempo necesario para diseñar y crear sus actividades docentes con el apoyo de recursos TIC.

2. Los profesores también manifiestan que los recursos TIC más utilizados para innovar en la metodología pedagógica universitaria acostumbran a ser los vídeos de YouTube, así como herramientas de participación colectiva como Kahoot.

3. Los profesores destacan su interés en participar y aprender en las formaciones para un uso avanzado de las TIC en la metodología pedagógica universitaria. 
En cuanto a la implementación en mayor grado de una metodología participativa y cooperativa de aprendizaje, podemos concluir los siguientes aspectos:

1. Los profesores universitarios que se formaron en Flipped Classroom aplicaron el nuevo modelo en sus aulas, aunque muchos solamente de modo parcial.

2. Los profesores universitarios que aplicaron parcialmente Flipped Classroom confirman la percepción de haberse actualizado y puesto al día en relación con los alumnos que tienen en clase.

3. Por su parte, los estudiantes mejoraron su rendimiento académico después de implementar el modelo Flipped Classroom en la Universidad Pontificia Comillas, aunque la mayor mejora se experimentó en proporción al grado de implicación de los estudiantes en cada una de las materias. Asimismo, es necesario destacar que los estudiantes no rechazaron de entrada el nuevo modelo formativo y se mostraban abiertos a explorar nuevos métodos de enseñanza-aprendizaje.

4. Relacionando las respuestas a diferentes preguntas, podemos llegar a la conclusión de que los profesores que participaron en las formaciones tienen una imagen positiva y eficaz del modelo Flipped Classroom como nuevo paradigma educativo, aunque destacan que aún les hace falta tiempo para diseñar de forma conjunta, debatir qué actividades pueden "dar la vuelta" y seleccionar qué herramientas tecnológicas son las más adecuadas para cada unidad. Así, y de forma resumida, podemos confirmar que las conclusiones más relevantes son:

1. El ritmo del cambio metodológico en la enseñanza superior con soporte TIC es lento y de naturaleza adaptativa. No es una cuestión meramente tecnológica (de herramientas, recursos, plataformas o software).

2. El profesorado universitario necesita espacios y tiempos para innovar metodológicamente. Exige tiempo, paciencia y acompañamiento al profesorado, lo que va más allá de unas horas limitadas de formación.

3. El alumnado universitario está preparado para cambiar de modelo formativo en la enseñanza superior y más si se utilizan las tecnologías digitales.

\section{Referencias}

Anguera, M.T. (2010). Complementariedad metodológica en la investigación en psicología: del enfren- tamiento al continuum. Jornadas de Psicología "Intervención psicológica en problemas sociales", Universidad Pontificia de Salamanca.

Bergmann, J., \& Sams, A. (2014). Flipped Learning: Gateway to Student Engagement, ISTE.

Bergmann, J., \& Sams, A. (2012). Flip your classroom: reach every student in every class every day. Eugene, OR.; Alexandria, VA.: ISTE ; ASCD.

Coll, C. (2010). Enseñar y aprender en el mundo actual: desafíos y encrucijadas. Pensamiento Iberoamericano, 7, 47-66. Recuperado de http://www.educacionysociedad.org/images/img_noticias/docu4e92a454ee17 8_10102011_452am.pdf

Delgado, C. (2014). Viajando a Ítaca por los mares cuantitativos, manual de ruta para investigar en grado y en postgrado. Salamanca: Amaru.

Fernández-Cruz, F. J., \& Fernández-Díaz, M. J. (2016). Los docentes de la Generación Z y sus competencias digitales [Generation Zs Teachers and their Digital Skills. Comunicar, 24(46), 97-105. doi: 10.3916. C462016-10.

González-Patiño, J., \& Esteban-Guitart, M. (2014). Some of the challenges and experiences of formal education in a Mobile-Centric Society (MCS). Digital Education Review, 25, 64-86.

Hernández Sampieri, R., \& Mendoza, C. P. (2008). El matrimonio cuantitativo cualitativo: el paradigma mixto. En J. L. Álvarez Gayou (presidente), 6 Congreso de Investigación en Sexología. Congreso efectuado por el Instituto Mexicano de Sexología, A. C. y la Universidad Juárez Autónoma de Tabasco, Villahermosa, Tabasco, México.

Hernández, R., Fernández, C., \& Baptista, P. (2014). Metodología de la Investigación (6 $6^{\mathrm{a}}$ ed.). México: McGraw-Hill.

Johnson, L., Adams Becker, S., Cummins, M., Estrada, V., Freeman, A., \& Hall, C. (2016). NMC Informe Horizon Edición Educación Superior 2016. Austin, Texas: The New Media Consortium.

Prats, M.A. \& Ojando, E.S. (2017). Guía para invertir tu clase paso a paso. Propuesta para un taller de formación. En J. Quintana \& O.Y. Aparicio (ed.), Temas emergentes en educación (pp. 101-115). Colombia: Universidad Central.

Schroer, W. J. (2008). Generations X, Y, Z and the others. The Journal of the Household Goods Forwarders Association of America, Inc, 40, 9-11.

Universidad de Comillas (2014). Plan estratégico 20142018, en https://www.comillas.edu/Documentos/ Plan_Estrategico_2014_2018.pdf 